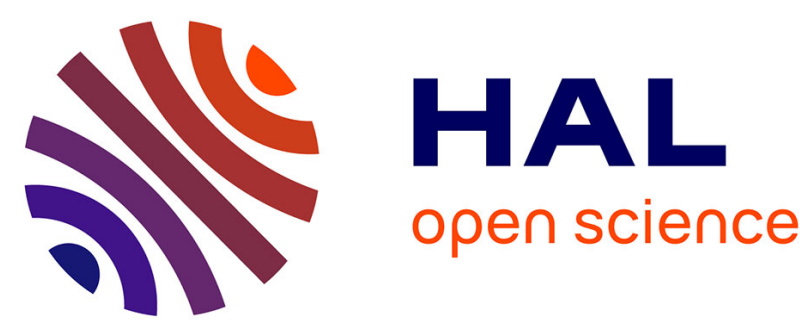

\title{
Lack of experimental evidence to support mcr-1- positive escherichia coli strain selection during oral administration of colistin at recommended and higher dose given by gavage in weaned piglets
}

Alexis Viel, Jérôme Henri, Agnès Perrin-Guyomard, Julian Laroche, William Couet, Nicolas Grégoire, Michel Laurentie

\section{To cite this version:}

Alexis Viel, Jérôme Henri, Agnès Perrin-Guyomard, Julian Laroche, William Couet, et al.. Lack of experimental evidence to support mcr-1- positive escherichia coli strain selection during oral administration of colistin at recommended and higher dose given by gavage in weaned piglets. International Journal of Antimicrobial Agents, 2017, 51 (1), pp.128-131. 10.1016/j.ijantimicag.2017.04.013 . anses01570163

\section{HAL Id: anses-01570163}

https://hal-anses.archives-ouvertes.fr/anses-01570163

Submitted on 22 Jun 2020

HAL is a multi-disciplinary open access archive for the deposit and dissemination of scientific research documents, whether they are published or not. The documents may come from teaching and research institutions in France or abroad, or from public or private research centers.
L'archive ouverte pluridisciplinaire HAL, est destinée au dépôt et à la diffusion de documents scientifiques de niveau recherche, publiés ou non, émanant des établissements d'enseignement et de recherche français ou étrangers, des laboratoires publics ou privés. 
$1 \quad$ Lack of experimental evidence to support mcr-1-positive Escherichia coli

2 strain selection during oral administration of colistin at recommended and

\section{8}

higher dose given by gavage in weaned piglets

anserm U1070, Pôle Biologie Santé - Bât. B36/37, 1 rue Georges Bonnet, Poitiers, France ${ }^{\mathrm{b}}$ Université de Poitiers, UFR Médecine-Pharmacie, 6 rue de la Milétrie, Poitiers, France

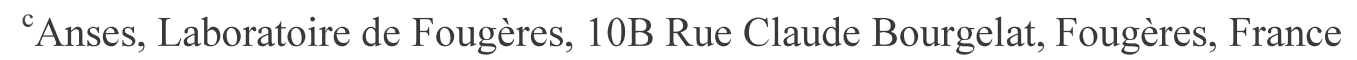
${ }^{\mathrm{d}}$ CHU Poitiers, 2 rue de la Milétrie, Poitiers, France Alexis Viel $^{\mathrm{a}, \mathrm{b}, \mathrm{c}}$, Jérôme Henric ${ }^{\mathrm{c}}$, Agnès Perrin-Guyomard ${ }^{\mathrm{c}}$, Julian Laroche ${ }^{\mathrm{a}, \mathrm{d}}$, William

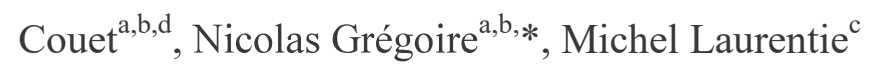

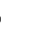

4

(1)
17

* Corresponding author

Tel.: +33 549366436 .

E-mail address: nicolas.gregoire@univ-poitiers.fr

\section{2}




\section{Abstract}

26 In this study, we assessed the selective effect of colistin orally administered to healthy weaned

27 piglets harbouring an intestinal $m c r-1$-positive Escherichia coli strain. Maximum recommended dose and a higher dose often used in European pig farms were given by gavage. No selection of the $m c r-1$-positive strain was observed in our controlled conditions whatever the dose. Further investigations in real farming conditions seem necessary.

Keywords: colistin; mcr-1; Escherichia col; piglets; selection 


\section{Introduction}

35 Colistin is an old polypeptidic antibiotic widely used in food-producing animals, especially in pig production as oral group treatment and metaphylaxis against Enterobacteriaceae digestive infections after weaning. Colistin is also used in human medicine as a last resort antibiotic against multi-drug bacteria. The first plasmid-mediated colistin resistance gene (mcr-1) discovered in China at the end of 2015 [1] has raised concern about the risk of spread of this resistance. Few months after, mcr-1 was detected in all continents, both in human and animals [2]. European Medicines Agency emphasized the need of reducing colistin use in animal and proposed to class colistin as critically important antimicrobials [3]. Scientific community lacks of in vivo data about $m c r-1$, especially in commensal Enterobacteriaceae to properly characterize the public health risk. We assessed here in piglets the selective effect of controlled colistin oral treatments on commensal intestinal Escherichia coli harbouring a $\mathrm{mcr}$ 1-positive strain with monitoring of faecal concentrations.

47 


\section{Materials and methods}

\subsection{Animals and housing}

Fifteen Large White-Landrace-Piétrain piglets were used to carry out the experiment, with no history of antimicrobials treatments. They were weaned at 21 days old and then fed with a standard non-medicated ration and had free access to water. After 5 days of collective housing, they were put in individual boxes with no possible contact ( 8 days before treatment, D-8).

\subsection{Bacterial strain and inoculation}

The original strain was a colistin-resistant $E$. coli $(\mathrm{MIC}=8 \mathrm{mg} / \mathrm{L})$ isolated from the intestines of a healthy pig sampled in a French slaughterhouse [4]. This strain harbouring $m c r-1$ (confirmed by PCR) was made rifampicin-resistant (MIC $>512 \mathrm{mg} / \mathrm{L}$ ) by spontaneous mutation before inoculation and named ECmcr1+. The inoculation phase consisted of three gavages (at D-7, D-5 and D-2) of $5 \mathrm{~mL}$ of about $10^{7} \mathrm{CFU} / \mathrm{mL}$ of ECmcr1+ in saline suspension.

\subsection{Experimental treatment and sampling}

Piglets were randomly divided into three groups of 5 animals and force-fed with colistin sulphate (Acti-coli, Biové, Arques, France) using a polyethylene tube, from D0 to D4 (5 days). RD group (for maximum Recommended Dosage) received 100000 UI/kg/day [5] equivalent to $3 \mathrm{mg} / \mathrm{kg} /$ day of colistin base activity (CBA) [6], given twice a day as $1.5 \mathrm{mg} / \mathrm{kg}$ in $5 \mathrm{~mL}$ solution; HD group (for Higher Dosage often found in pig farms [7]) received $200000 \mathrm{UI} / \mathrm{kg} /$ day equivalent to $6 \mathrm{mg} / \mathrm{kg} /$ day of CBA, given twice a day as $3 \mathrm{mg} / \mathrm{kg}$ in $5 \mathrm{~mL}$ solution; the placebo group received water. Fresh faecal samples were taken on mornings (after anal stimulation) from the day before ECmcr1+ inoculation (D-8) until 19 days after the 
end of colistin treatment (D23). This experiment was approved by the ComEth Anses/ENVA/UPEC n 16 (French ethical committee) under the reference APAFIS\#29052015112717486085.

\subsection{Microbiological analysis and colistin assay}

About $1 \mathrm{~g}$ of each fresh faecal sample was diluted in saline solution. Selected dilutions were plated on Mac Conkey agar (BD, Le Pont de Claix, France) alone or supplemented with 200 $\mathrm{mg} / \mathrm{L}$ of rifampicin (Sigma, Saint-Quentin Fallavier, France) in order to count total E. coli and ECmor1+, respectively.

Another specimen ( $1 \mathrm{~g}$ of faeces) from D0 to D9 was kept at $-20^{\circ} \mathrm{C}$ until colistin was assayed by a LC-MS/MS method adapted from previous works $[8,9]$. Briefly, faeces were mixed with blank plasma and diluted in $10 \mathrm{~mL}$ of acetonitrile with $6 \%$ of trichloroacetic acid. After vortexing, centrifugation and evaporation, dry matter was diluted in buffer $(\mathrm{pH}=7.2)$ with blank plasma and loaded on Oasis HLB Catridges (Waters, Milford, MA, USA). After washing and eluates evaporation, residues were analysed by HPLC-MS/MS with a limit of quantification (LOQ) of $1 \mu \mathrm{g} / \mathrm{g}$ of faeces. Quality controls were prepared at 2.5, 12.5 and 18.8 $\mu \mathrm{g} / \mathrm{g}$ of faeces. a Student-T test. Mean values of total faecal E. coli and of ECmcr1+ for treated groups were compared to those of the placebo group using a Student-T test. All statistical analyses were carried out using R 3.3.2 [10]. 


\section{Results}

High faecal colistin concentrations were measured with mean values greater than or equal to about $200 \mu \mathrm{g} / \mathrm{g}$ of faeces from D2 to D5 in the two treated-groups (Table 1). No significant differences were observed between these groups (T-test) due to the high inter-individual variability. Three days after the end of treatment (D7), faecal concentrations were reduced by about 100-fold and felt below the LOQ after 2 days more (D9).

No significant differences were found for total faecal E. coli (ECtot) population between groups before colistin administration (from D-8 to D0, Fig. 1A). We observed a slight but non-significant decrease of ECtot during the treatment phase for the placebo group.

Conversely, a stronger reduction of ECtot was noticed for each colistin-treated group between D0 and D3 but only significant for HD group (compared to placebo, Fig. 1A). Then, a return to initial level of ECtot was observed 2 and 3 days after last colistin dose (D4) for RD and HD group, respectively.

After the inoculation phase (D-7 to D-2), ECmcr1+ reached up to $2 \%$ of ECtot (Fig. 1B). However 7 of 15 piglets had ECmcr1+ count lower to the LOQ (1.2 log CFU/g of faeces) just before treatment; for 5 of them the strain was undetectable during all the experiment ( 2 in HD group, 2 in placebo group and 1 in RD group). Mean ECmcr1+ levels were equivalent between the three groups before the first colistin administration (D0). Overall, ECmcr1+ level compared favourably between each group i.e. remained relatively constant during and after treatment. 


\section{Discussion}

122 To our knowledge, this is the first in vivo study exploring the selective effect of colistin on

123 $m c r-1$-positive $E$. coli in pigs. Previous studies already attested a rare emergence of colistinresistant E. coli after oral colistin treatment of healthy [7] and sick piglets [11], therefore we neglected it. Exogenous resistant bacteria inoculation in treated pigs already supported the selective effect of other antimicrobials in these animals [12]. In order to control the colistin doses, we chose to force-feed the piglets for treatment. The absence of significant differences of faecal colistin concentrations between $\mathrm{HD}$ and $\mathrm{RD}$ groups was likely due to measurement uncertainty. However, these results seemed relevant: with a daily faecal excretion of piglets of around $250 \mathrm{~g}$ [13], we can estimate that about 60 and $95 \%$ of the colistin dose was recovered in faeces for HD and RD group. This is consistent with the poor absorption of colistin sulphate after oral treatments in pigs [5]. In addition, we chose to induce a rifampicin resistance in ECmcr1+ in order to monitor precisely this strain and due to the poor accuracy of colistin-supplemented media for resistant Enterobacteriaceae isolation [7, 11].

The initial decrease of ECtot level before treatment and the slight continuous one of placebo group were probably due to the weaning process that disturbed the microbiota equilibrium [14]. The high colistin treatment induced a significant reduction of ECtot counts compared to the placebo group (Fig. 1A), but the recommended dose poorly affected it. Considering the high faecal concentrations during the treatment phase $(>200 \mu \mathrm{g} / \mathrm{g})$, greater reduction of $E$. coli would be expected. Colistin high adsorption to faecal fibres could lead to a dramatic decrease of its effect [15]. In addition, for polymyxin B, up to $90 \%$ of the initial dose could be reversibly bound to faeces [16]. Although this was shown with a bioassay method and not with colistin, the real active colistin concentrations in our experiment should be greater than $20 \mu \mathrm{g} / \mathrm{g}$ of faeces. This is normally still enough to reduce ECtot population as it is composed 
of majority of colistin-sensitive strains $(\mathrm{CMI} \leq 2 \mathrm{mg} / \mathrm{L})$. Therefore, the absence of significant difference of the ECtot evolution between the two treated groups is still unclear. The small number of animal per group is perhaps a limiting factor. Moreover, inter and intra-individual variabilities of faecal colistin concentrations were high, probably due to a heterogeneous colistin distribution within faeces. Therefore, the actual effective concentrations of colistin, to which bacteria were exposed within the digestive tract, are mostly unknown.

In the placebo group, the counts of ECmcr1+ were low but remained stable enough over the experiment (Fig. 1B). No selective effect of colistin in treated piglets was observed as ECmcr1+ stayed at a constant level over time, whatever the dose. This suggests that, in standardized conditions, bacteria were exposed during a too short period to concentrations within the selection window, i.e. between the MICs of indigenous E. coli and of ECmcr1+ $(8 \mathrm{mg} / \mathrm{L})$. In comparison, when colistin is administered on a large scale through water or feed on pig farms, colistin concentration in faeces are lower [7] and much more variable [17]; therefore the probability to select strains harbouring $m c r-1$ increases. Despite this, no real outbreak of $m c r-1$ positive strains exists in pig production in Europe [2] where a low prevalence of $m c r-1$ positive E. coli is found (e.g. $0.5 \%$ in French pig farms) [4]. In contrast, a high prevalence is observed in Asia (more than $20 \%$ in China) [2], where colistin has been used for decades as growth-promoter for piglets [18]. This sub-therapeutic dose (about 4-5 times lower than therapeutic use [19]) administered during a long period is likely to give digestive concentrations reaching the selection window of $m c r-1$ positive strains. Awareness of this high risk led China to ban colistin as promoter very recently [20]. This hypothesis should deserve further considerations. 
172 In conclusion, under this experimental setting in piglets, oral dosing with colistin did not

173 induce selection of $m c r-1$ positive strains. Further investigations should be necessary to

174 confirm this observation in farming conditions (with colistin given via food or water and

175 piglets having contact between them). Meanwhile, a responsible and careful use of colistin is

176 required to preserve this last-resort antimicrobial agent.

Acknowledgments

179

The authors would like to thank Jean-Guy Rolland and Mireille Bruneau, Anne De Courville, Karine Deleurme, Pamela Houée, Catherine Poirier for their technical assistance.

\section{Declarations}

Funding: Alexis Viel was supported by a doctoral fellowship from the French National

Institute of Health and Medical Research (Inserm) and the French Agency for Food,

185 Environmental and Occupational Health \& Safety (Anses).

Competing Interests: None

Ethical Approval: This experiment was approved by the ComEth Anses/ENVA/UPEC n 16 


\section{References}

[1] Liu Y-Y, Wang Y, Walsh TR, Yi L-X, Zhang R, Spencer J, et al. Emergence of plasmidmediated colistin resistance mechanism MCR-1 in animals and human beings in China: a microbiological and molecular biological study. The Lancet infectious diseases. 2016;16:1618.

[2] Skov R, Monnet D. Plasmid-mediated colistin resistance (mcr-1 gene): three months later, the story unfolds. Euro surveillance : bulletin Europeen sur les maladies transmissibles = European communicable disease bulletin. 2016;21:30155.

[3] Updated advice on the use of colistin products in animals within the European Union: development of resistance and possible impact on human and animal health.: European Medicines Agency (EMA); 2016.

[4] Perrin-Guyomard A, Bruneau M, Houée P, Deleurme K, Legrandois P, Poirier C, et al. Prevalence of mcr-1 in commensal Escherichia coli from French livestock, 2007 to 2014.

Euro surveillance : bulletin Europeen sur les maladies transmissibles = European communicable disease bulletin. 2016;21.

[5] Guyonnet J, Manco B, Baduel L, Kaltsatos V, Aliabadi M, Lees P. Determination of a dosage regimen of colistin by pharmacokinetic/pharmacodynamic integration and modeling for treatment of GIT disease in pigs. Research in veterinary science. 2010;88:307-14.

[6] Nation RL, Li J, Cars O, Couet W, Dudley MN, Kaye KS, et al. Consistent Global Approach on Reporting of Colistin Doses to Promote Safe and Effective Use. Clinical Infectious Diseases. 2014;58:139-41.

[7] Fleury MA, Jouy E, Eono F, Cariolet R, Couet W, Gobin P, et al. Impact of two different colistin dosing strategies on healthy piglet fecal microbiota. Research in Veterinary Science. 2016;107:152-60. 
[8] Van den Meersche T, Van Pamel E, Van Poucke C, Herman L, Heyndrickx M, Rasschaert

$\mathrm{G}$, et al. Development, validation and application of an ultra high performance liquid chromatographic-tandem mass spectrometric method for the simultaneous detection and quantification of five different classes of veterinary antibiotics in swine manure. Journal of Chromatography A. 2016;1429:248-57.

[9] Gobin P, Lemaitre F, Marchand S, Couet W, Olivier JC. Assay of colistin and colistin methanesulfonate in plasma and urine by liquid chromatography-tandem mass spectrometry. Antimicrobial agents and chemotherapy. 2010;54:1941.

[10] R Core Team. R: A language and environment for statistical computing. R Foundation for Statistical Computing,Vienna, Austria; 2016.

[11] Rhouma M, Beaudry F, Thériault W, Bergeron N, Beauchamp G, Laurent-Lewandowski

S, et al. In vivo therapeutic efficacy and pharmacokinetics of colistin sulfate in an experimental model of enterotoxigenic Escherichia coli infection in weaned pigs. Veterinary Research. 2016;47:58.

[12] Cavaco LM, Abatih E, Aarestrup FM, Guardabassi L. Selection and Persistence of CTX-

M-Producing Escherichia coli in the Intestinal Flora of Pigs Treated with Amoxicillin,

Ceftiofur, or Cefquinome. Antimicrobial Agents and Chemotherapy. 2008;52:3612-6.

[13] Pouliot F, Godbout S, Dufour V, Vob Bernuth R, Hill J. Évaluation de l'efficacité d'un système de séparation fèces-urine sous caillebotis en engraissement: bilan de masse et caractérisation des sous-produits. Journées Rech Porcine. 2005;37:45-50.

[14] Swords WE, Wu C-C, Champlin FR, Buddington RK. Postnatal changes in selected bacterial groups of the pig colonic microflora. Neonatology. 1993;63:191-200.

[15] Van Saene JJ, Van Saene HK, Stoutenbeek CP, Lerk CF. Influence of faeces on the activity of antimicrobial agents used for decontamination of the alimentary canal. 
241 [16] Hazenberg M, Pennock-Schröder A, Van de Merwe J. Reversible binding of polymyxin

242 B and neomycin to the solid part of faeces. Journal of Antimicrobial Chemotherapy.

$243 \quad 1986 ; 17: 333-9$.

244 [17] Soraci AL, Amanto F, Tapia MO, de la Torre E, Toutain P-L. Exposure variability of

245 fosfomycin administered to pigs in food or water: impact of social rank. Research in

246 veterinary science. 2014;96:153-9.

247 [18] Kim DP, Saegerman C, Douny C, Dinh TV, Xuan BH, Vu BD, et al. First survey on the 248 use of antibiotics in pig and poultry production in the Red River Delta region of Vietnam.

249 Food and Public Health. 2013;3:247-56.

250 [19] Rhouma M, Beaudry F, Letellier A. Resistance to colistin: what is the fate for this 251 antibiotic in pig production? International journal of antimicrobial agents. 2016;48:119-26.

252 [20] Walsh TR, Wu Y. China bans colistin as a feed additive for animals. The Lancet 253 Infectious Diseases. 2016;16:1102-3. 
255 Figure Legend

256 Fig 1: Mean ( \pm SD) of counts of total faecal E.coli (A) and ECmcr1+ (B) before, during and 257 after oral colistin (or water) administrations for HD group (green), RD group (blue) and 258 placebo group (red). Vertical arrows indicate ECmcr1+ inoculations. The vertical dotted lines 259 indicate the treatment period (D0 to D4). Horizontal dashed line represents the limit of 260 quantification $(\log 1.2)$ and data below this value were arbitrary put to value log 0.6. Similar 261 profiles were obtained when data below LOQ were fixed at zero or at LOQ. Significant differences from placebo group with Student test: * $(\mathrm{p}<0.05) ; * *(\mathrm{p}<0.01)$ 


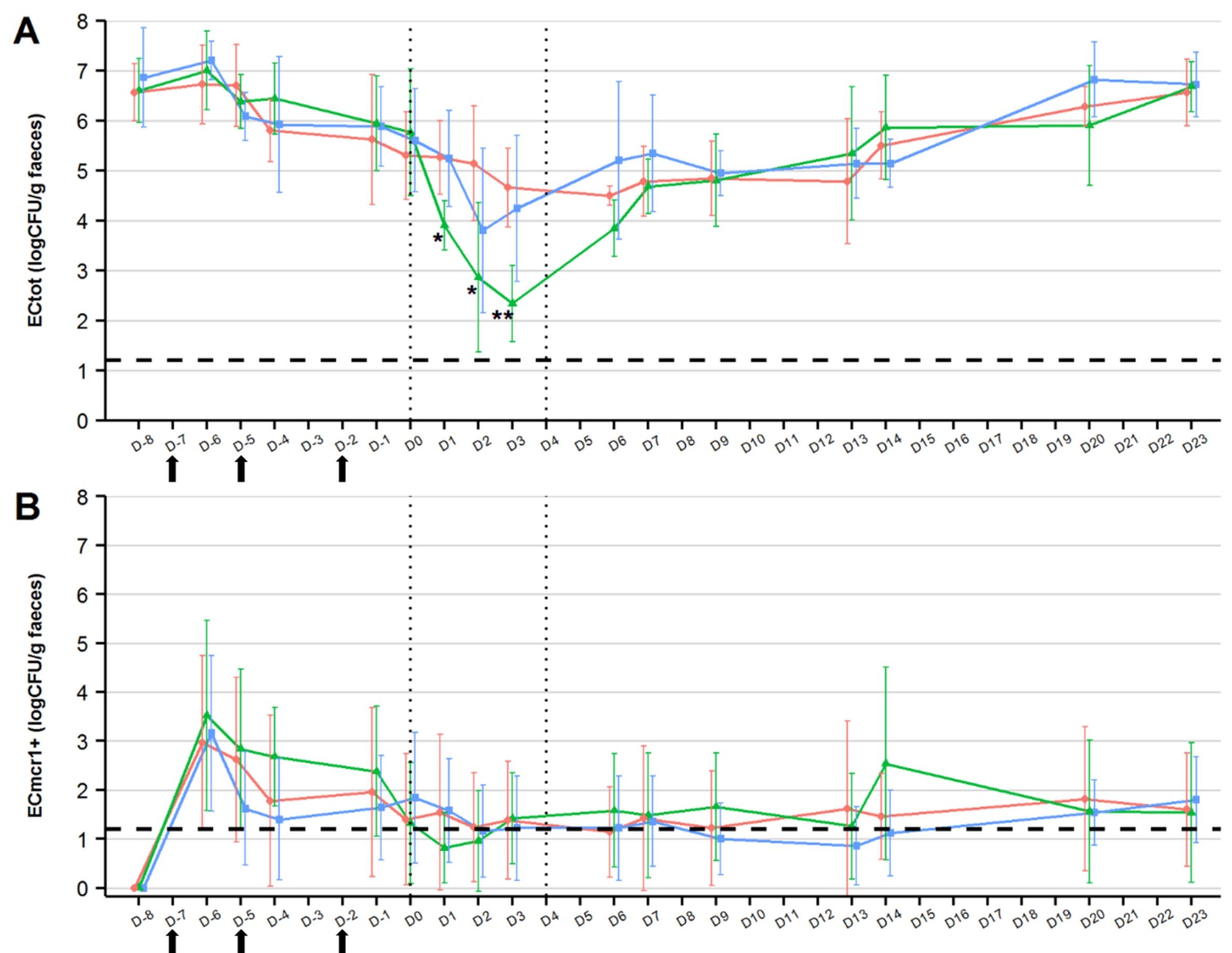




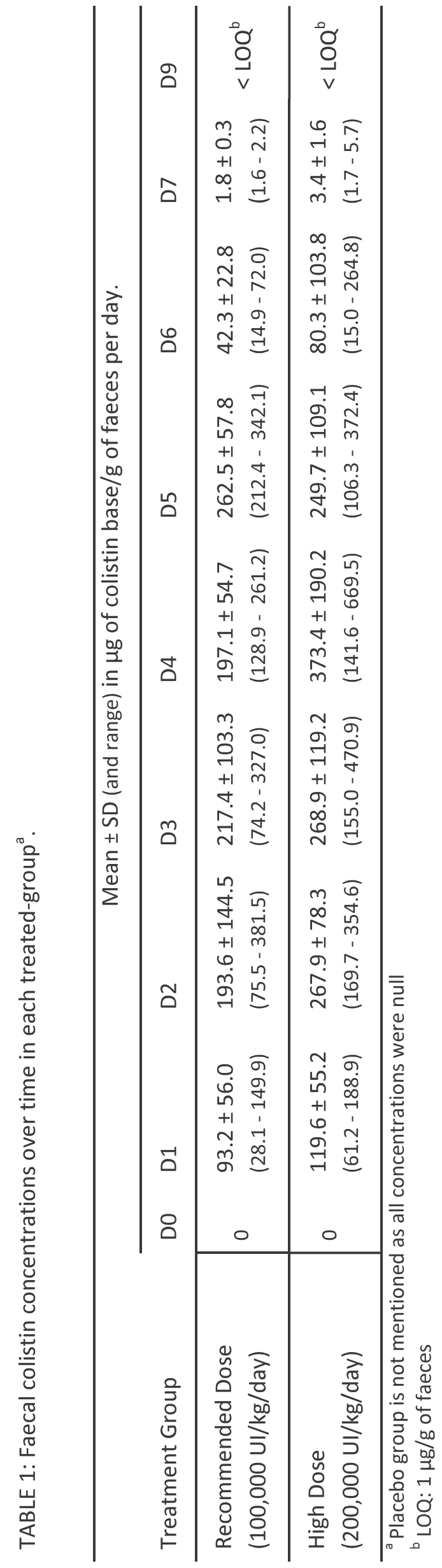

